\title{
Spatio-temporal-based joint range and angle estimation for wideband signals
}

\author{
Guilhem Villemin*, Caroline Fossati and Salah Bourennane
}

\begin{abstract}
Object localization using active sensor network exploiting the scattering of the emitted waves by a transmitter has been drawing a lot of research interest in the last years. For most applications, the environment leads to the arrival of multiple signals corresponding to emitted signal, signals which are scattered by the objects, and noise. In practical systems, the signals impinging on an array are frequently correlated, and the object number rapidly exceeds the number of sensors, making unsuitable most high-resolution methods used in array processing. We propose a solution to overcome these two experimental constraints. Firstly, frequential smoothing is used to decorrelate the scattered signals, enabling the estimation of their time delays of arrival (TDOA), using subspace-based methods. Secondly, an efficient algorithm for source localization using the TDOA is proposed. The advantage of the developed method is its efficiency even if the number of sources is larger than the number of sensors, in the presence of correlated signals. The performances of the proposed method are assessed on simulated signals. The results on real-world data are also presented and analyzed.
\end{abstract}

Keywords: Source localization; Wideband signals; Time delay estimation; High-resolution algorithm; Correlation

\section{Introduction}

Detection and localization of scattering objects located entirely above or below a surface, which has many applications in a number of fields, turn out to be very important today. In the Earth sciences, it is used to study bedrock, soils, groundwater and ice. In archaeology, it is used in law enforcement, for locating wreckage, mapping archaeological ruins, clandestine graves and buried evidences. The civil applications include detecting buried services under city streets (pipes, cables...), continuous inspection of layers in road pavements and airport runways, mapping cavities or voids beneath road pavements, runways or behind tunnel linings, monitor the condition of railway ballast, and detect zones of clay fouling leading to track instability. Over the past few decades, a significant amount of research effort has been spent towards developing a viable buried object detection scheme. Several electromagnetic wave methods, for example, groundpenetrating radar (GPR, sometimes called georadar or subsurface radar), have proved that EM can give a good

*Correspondence: guilhem.villemin@fresnel.fr

CNRS-UMR 7249/Fresnel Institute, Ecole Centrale Marseille, Aix-Marseille Université, D.U. de Saint-Jérôme, Marseille Cedex 20 13397, France performance in buried object detection [1,2], particularly using high-resolution methods [3]. However, the depth range of GPR suffers from many limitation.

Recently, it was performed using both acoustical waves and array processing algorithms in order to improve depth range and spatial resolution. Usually, the parameters of interest are the directions of arrival (DOA) of the radiating objects and their range from the array. Conventional beamforming offers a limited spatial resolution, and this has led to the development and successful application of more advanced techniques. Examples are Capon's minimum variance method [4], and a variety of methods based on eigendecomposition, such as multiple signal classification (MUSIC) [5].

These high-resolution subspace-based methods for DOA estimation, essentially based on the spatial diversity induced by a great number of sensors, giving enough information to address the DOA estimation issue, are well adapted to narrowband signals. High-resolution subspace-based methods have also been extended to the wideband signals. Many methods have been proposed to estimate the DOA problem of wideband sources [6-13]. Among these methods, incoherent subspace methods $[7,8]$ were proposed firstly. They estimate the DOAs

\section{Springer}

(c) 2013 Villemin et al.; licensee Springer. This is an Open Access article distributed under the terms of the Creative Commons Attribution License (http://creativecommons.org/licenses/by/2.0), which permits unrestricted use, distribution, and reproduction in any medium, provided the original work is properly cited. 
of wideband sources separately at each frequency bin and then combine the results obtained at each frequency to get a final estimate. High-resolution methods simply need to meet the following assumptions: a linear equispaced array including at least one more sensor than radiating sources, white and Gaussian background noise spatially uncorrelated and uncorrelated signals of the different sources. It is important to note that in practice, these assumptions are obviously rarely all fulfilled [14-16], especially the last one.

To overcome this drawback, Wang and Kaveh [8] proposed a coherent signal-subspace method. In this method, the covariance matrices of different frequency bins are focused by proper transformation matrices and averaged to create a universal matrix. Then, a high-resolution narrowband method, such as MUSIC, could be applied to estimate the DOAs. Subsequently, many improved methods have been proposed to design a new focusing matrix without focusing loss or with smaller bias, such as rotational signal-subspace [9], two-sided correlation transformation [10] and so on.

Although these focusing methods decrease the resolution threshold and reduce the estimation bias, their performance greatly depends on the accuracy of the initial angles. Another wideband DOA algorithm named test of orthogonality of projected subspaces was proposed [11]. It does not need initial angles and can show better performance at mid-signal-to-noise ratio (SNR). However, it cannot avoid false peaks in the spatial spectrum.

All the signal-subspace methods mentioned above have a common constraint that the number of sources should be less than the number of sensors. Lately, a KhatriRao subspace approach [13] was proposed, whose major advantage is that it can perform well even if the number of sensors is about half of the number of sources. However, it depends on quasi-stationary sources and needs a large amount of snapshots to obtain a satisfying performance. Moreover, needing always more sensors than sources raises several problems in buried objects localization. For instance, the cost and length of the antenna needed to support a great number of sensors.

In this paper, we propose to overcome the problem of the number of sensors in the case of wideband signals, addressing the problem for all the frequency band at each sensor. The spatial diversity induced by the great number of sensors (used in classical methods) will be here replaced for each sensor by the frequential diversity of the broadband signals. It is proposed to divide the frequency band of each data recorded on each sensor into frequency sub-bands. After applying a smoothing algorithm [3,17-20] on these sub-bands, it is possible to apply a subspace-based method that will give information about the different TDOA of the signals recorded on each sensor. Identifying as many sets of TDOA as sources enables us to estimate their range and DOA.
The remainder of the paper is as follows. The 'Overview of localization methods' section briefly presents some classical array processing methods. The 'High-resolution algorithm for wideband signals in time domain' section proposes an adaptation of the high-resolution algorithm for wideband signal using frequency diversity on each sensor instead of the array spatial diversity, a frequential smoothing method is described and a whitening procedure of the signals is also proposed to improve the method. The 'Source localization' section deals with the source localization issue. Finally, the 'Main algorithm' and 'Numerical results' sections present the main algorithm and some results obtained on simulated and real data.

In this paper, the superscript ' $T$ ' represents transpose operator, superscript ' + ' denotes conjugate transpose operator, superscript ' $*$ ' represents conjugate operator and $E[$.$] denotes the mathematical expectation.$

\section{Overview of localization methods Signal model}

Consider an array of $N$ sensors which receive the signals in one wave field generated by the scattering of one emitted signal by $P,(P<N)$ objects, which further will be called sources, in the presence of an additive noise [21], see Figure 1. The received signal vector is sampled and the fast Fourier transform algorithm (FFT) is used to compute the discrete Fourier transform (DFT). The array outputs are represented by:

$$
\mathbf{r}(f)=\mathbf{A}(f, \boldsymbol{\theta}) \mathbf{s}(f)+\mathbf{n}(f),
$$

where $\mathbf{r}(f), \mathbf{s}(f)$ and $\mathbf{n}(f)$ are, respectively, the DFT of the array outputs, the source signals and the noise vectors. Matrix $\mathbf{A}(f, \boldsymbol{\theta})$, of dimensions $(N \times P)$, is the transfer matrix of the source-sensor array system, and $\boldsymbol{\theta}=$ $\left[\theta_{1}, y \cdots, \theta_{P}\right]^{T}$ is a vector containing the DOA of the sources.

$$
\begin{aligned}
\mathbf{A}(f, \boldsymbol{\theta}) & =\left[\mathbf{a}\left(f, \theta_{1}\right) \cdots \mathbf{a}\left(f, \theta_{P}\right)\right], \text { where } \\
\mathbf{a}(f, \boldsymbol{\theta}) & =\left[1, e^{-i \phi_{\theta}}, \cdots, e^{-i(N-1) \phi_{\theta}}\right]^{T}
\end{aligned}
$$

and $\phi_{\theta}=\frac{-2 \pi f d \sin (\theta)}{v} . v$ is the velocity of the wave, and $d$ the distance between two consecutive sensors. The sensor noises are assumed to be independent of the source signals and spatially correlated. The covariance matrix of the data can be defined by the $(N \times N)$ matrix:

$$
\boldsymbol{\Gamma}(f)=E\left[\mathbf{r}(f) \mathbf{r}^{+}(f)\right]=\mathbf{A}(f) \boldsymbol{\Gamma}_{s}(f) \mathbf{A}^{+}(f)+\boldsymbol{\Gamma}_{n}(f),
$$

where $\boldsymbol{\Gamma}_{n}(f)=E\left[\mathbf{n}(f) \mathbf{n}^{+}(f)\right]$ is the $(N \times N)$ noise covariance matrix, and $\boldsymbol{\Gamma}_{s}(f)=E\left[\mathbf{s}(f) \mathbf{s}^{+}(f)\right]$ is the $(P \times P)$ source signals covariance matrix.

In the following, we present a high-resolution source localization method exploiting algebraic properties of the covariance matrix $\boldsymbol{\Gamma}(f)$. 


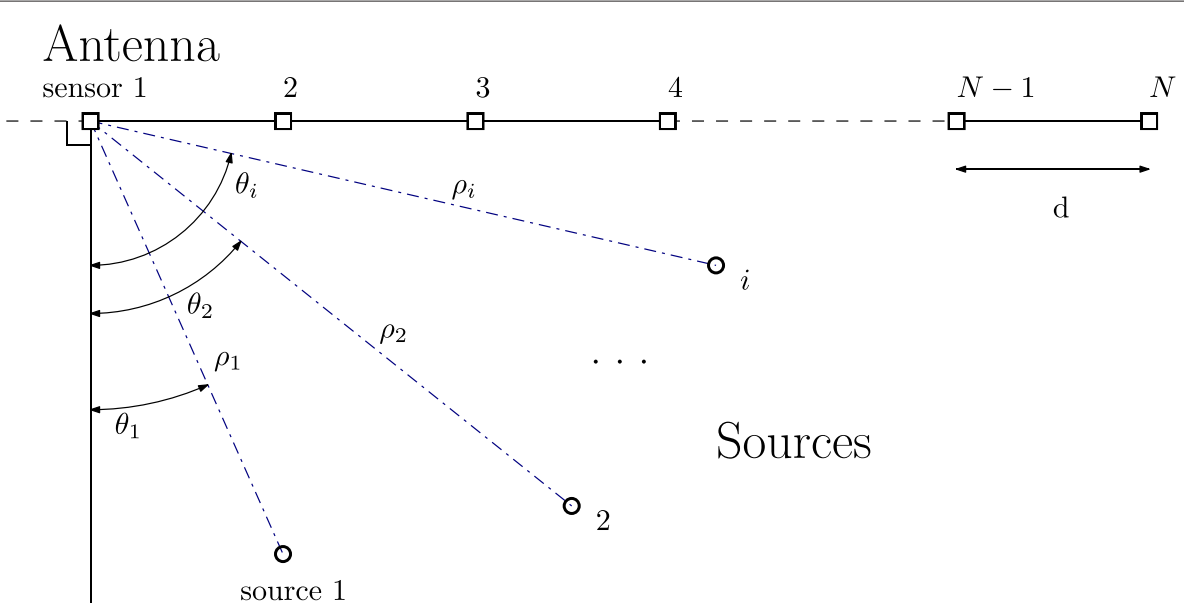

Figure 1 A linear equispaced array with several sources. The first sensor of the array is used as a reference for the sources' ranges and DOAs.

\section{High-resolution methods}

The high-resolution methods exploit the statistics of the recorded signals [21-23]. The principle is to exploit the structure of the vector space which is spanned by the measures collected upon the sensors. This vector space is the direct sum of the source signal subspace and the noise subspace. These methods are efficient when $\boldsymbol{\Gamma}_{s}(f)$ is full rank, i.e. when the signals are decorrelated. The signal subspace is spanned by the eigenvectors associated with the $P$ largest eigenvalues, the noise subspace is spanned by eigenvectors associated with the $N-P$ smallest eigenvalues. Thus, the covariance matrix can be written:

$$
\boldsymbol{\Gamma}(f)=\left[\mathbf{V}_{s}(f) \mathbf{V}_{n}(f)\right]\left[\begin{array}{cc}
\boldsymbol{\Psi}_{s}(f) & 0 \\
0 & \boldsymbol{\Psi}_{n}(f)
\end{array}\right]\left[\mathbf{V}_{s}(f) \mathbf{V}_{n}(f)\right]^{+}
$$

where $\mathbf{V}_{s}(f)$ and $\mathbf{V}_{n}(f)$ are the matrices containing the eigenvectors associated with the signal and the noise subspace, respectively, $\boldsymbol{\Psi}_{s}$ and $\boldsymbol{\Psi}_{n}$ are diagonal matrices containing eigenvalues associated with the signal and noise subspaces.

Multiple signal classification (MUSIC) is the best known high-resolution method. It exploits the orthogonality between the signal subspace and the noise subspace. The DOA of sources is given by the positions of the maxima of the pseudo-spectrum represented by:

$$
F_{\operatorname{MUSIC}}(f, \theta)=\frac{1}{\mathbf{a}^{+}(f, \theta) \mathbf{V}_{n}(f) \mathbf{V}_{n}^{+}(f) \mathbf{a}(f, \theta)},
$$

where $\theta \in\left[-90^{\circ}, 90^{\circ}\right]$.

The implementation of MUSIC requires the eigendecomposition of the covariance matrix $\boldsymbol{\Gamma}(f)$. The conventional methods are achieved by either the eigenvalue decomposition or the singular values decomposition (SVD). However, the main drawback of this conventional decomposition is its inherent important computational load. Indeed, the number of sensors $N$ is often larger than the number of sources $P$. It means that the dimension of the noise subspace $(N-P)$ is often larger than the signal subspace dimension $(P)$. It is more efficient to use solely the signal subspace than the noise subspace. Indeed, we can calculate the signal subspace $\mathbf{V}_{s}(f)=\left[\mathbf{v}_{1}(f), \mathbf{v}_{2}(f), \ldots, \mathbf{v}_{P}(f)\right]$ whose columns are the $P$ orthonormal basis vectors. The projector onto the noise subspace spanned by the $(N-P)$ eigenvectors associated with the $(N-P)$ smallest eigenvalues is $\mathbf{V}_{n}(f) \mathbf{V}_{n}^{+}(f)$ and can be given by:

$$
\mathbf{V}_{n}(f) \mathbf{V}_{n}^{+}(f)=\mathbf{I}-\mathbf{V}_{s}(f) \mathbf{V}_{s}^{+}(f),
$$

where $\mathbf{I}$ is the identity matrix.

\section{High-resolution algorithm for wideband signals in time domain \\ Proposed model}

When the number of sensors is smaller than the number of sources, we propose to exploit the bandwidth of the source signals, using a high-resolution algorithm to estimate the TDOA of the signals received on each sensor. The spectral information received on each sensor is divided into a number of frequencies $M$ larger than the number of sources $P, P<M$. These frequencies will play in the high-resolution algorithm the same role as the sensors in the classical array processing methods.

Consider a sensor $j$ which receives the scattered signals $s$ generated by $P$ objects in the presence of an additive noise. The signal received on sensor $j$ can be written as:

$$
r_{j}(t)=\sum_{i=1}^{P} c_{i, j} s\left(t-\tau_{i, j}\right)+n_{j}(t),
$$


where $c_{i, j}$ represents an amplitude and phase shift term and is assumed to be independent of time, $\tau_{i, j}$ stands for the $(i, j)$ th TDOA and $n_{j}$ is an additive noise. The Fourier transform of $r_{j}(t)$ is

$$
\tilde{r}_{j}(f)=\sum_{i=1}^{P} c_{i, j} \tilde{s}(f) e^{-2 i \pi f \tau_{i, j}}+\tilde{n}_{j}(f)
$$

as the signal is sampled, the FFT is used to compute the DFT. Further, this representation will be used:

$$
\tilde{\mathbf{r}}_{j}=\Lambda \mathbf{A}_{j} \mathbf{c}_{j}+\tilde{\mathbf{n}}_{j},
$$

where $\tilde{\mathbf{r}}_{j}=\left[\tilde{r}_{j}\left(f_{1}\right), \cdots, \tilde{r}_{j}\left(f_{m}\right), \cdots, \tilde{r}_{j}\left(f_{M}\right)\right]^{T}$ is the DFT of the sensor output, $\boldsymbol{\Lambda}=\operatorname{diag}\left(\tilde{s}\left(f_{1}\right), \cdots, \tilde{s}\left(f_{m}\right), \cdots, \tilde{s}\left(f_{M}\right)\right)$ is the known diagonal matrix made of the signal Fourier transform, $\mathbf{c}_{j}=\left[c_{1, j}, \cdots, c_{i, j}, \cdots, c_{P, j}\right]^{T}$ is the vector of the $c_{i, j}$ and $\tilde{\mathbf{n}}_{j}=\left[\tilde{n}_{j}\left(f_{1}\right), \cdots, \tilde{n}_{j}\left(f_{m}\right), \cdots, \tilde{n}_{j}\left(f_{M}\right)\right]^{T}$ is the vector of the noise DFT. The $(M \times P)$ matrix $\mathbf{A}_{j}$ is the transfer matrix of the source-frequency system with respect to some chosen reference times. $\mathbf{A}_{j}=\left[\mathbf{a}\left(\tau_{1, j}\right) \cdots \mathbf{a}\left(\tau_{P, j}\right)\right]$, where $\mathbf{a}(\bullet)=\left[e^{-2 i \pi f_{1}(\bullet)}, e^{-2 i \pi f_{2}(\bullet)}, \cdots, e^{-2 i \pi f_{M}(\bullet)}\right]^{T}$.

The sensor noise is assumed to be independent of the source signals. On each sensor, the high-resolution algorithms can be used to estimate the different TDOA. Using the source TDOA sets estimated on the different sensors allows us to localize the sources. As the proposed method is applied to all sensors independently and the obtained TDOA are simultaneously used to localize the sources, we will get rid of the subscript $j$ to simplify the notations. In the following section, we present the proposed method.

\section{TDOA estimation for a given sensor $\boldsymbol{j}$}

As in Eq. (3), the covariance matrix of the data can be defined by the $(M \times M)$-dimensional matrix:

$$
\boldsymbol{\Gamma}=E\left[\tilde{\mathbf{r}} \tilde{\mathbf{r}}^{+}\right] .
$$

As the noise and the signal are assumed to be independent,

$$
\boldsymbol{\Gamma}=\boldsymbol{\Lambda} \mathbf{A} \boldsymbol{\Gamma}_{c} \mathbf{A}^{+} \boldsymbol{\Lambda}^{+}+\boldsymbol{\Gamma}_{\tilde{n}},
$$

where $\boldsymbol{\Gamma}_{\tilde{n}}$ is the $(M \times M)$ noise covariance matrix and $\boldsymbol{\Gamma}_{c}=$ $E\left[\mathbf{c c}^{+}\right]$.

$$
\begin{aligned}
& \text { Let } \mathbf{A}^{\prime}=\boldsymbol{\Lambda} \mathbf{A} \text { and } \\
& \qquad \boldsymbol{\Gamma}=\mathbf{A}^{\prime} \boldsymbol{\Gamma}_{c} \mathbf{A}^{\prime+}+\boldsymbol{\Gamma}_{\tilde{n}}
\end{aligned}
$$

This data model allows to use high-resolution algorithms of array processing on the matrix $\boldsymbol{\Gamma}$ using $\mathbf{a}^{\prime}=\mathbf{\Lambda} \mathbf{a}$ instead of a to extract the TDOA on each sensor.

In this paper, we assume that $P$ is known or can be estimated, for instance, by sorting the eigenvalues of $\boldsymbol{\Gamma}$ or using the known criteria AIC and MDL [24,25].

Although the high-resolution algorithms assume that the matrix $\Gamma$ is full rank, this assumption is not fulfilled, due to the fact that we are dealing in this paper with $P$ totally correlated signals.

\section{Frequential smoothing}

If the matrix $\Gamma$ is not full rank, which is the case in the considered problem, the performances of the high-resolution algorithms will be degraded. The SVD will not be relevant enough and the signal subspace will be under-estimated [8-10]. For instance, some eigenvectors will be lost to describe this subspace.

To avoid this problem, spatial and frequential smoothing methods are proposed [8-10,20]. Their efficiency relies on the number of sensors or on the frequency bandwith of the signals, respectively [3,17-20]. In this paper, we address the following issue: only few sensors are available and the source signals are totally correlated signals. That is why we propose to use a frequential smoothing method. The method estimates an unbiased covariance matrix of the observation and reduces the signal correlation [20]. The modified spatial smoothing processing (MSSP) method exploits the translation invariance and the backward propagation to estimate the covariance matrix.

The frequency band of $M$ frequencies is divided into $K$ sub-bands of $L$ frequencies with a certain overlap, as shown in Figure 2. Usually, the maximum overlap between two consecutive sub-bands is $L-1$ frequencies, yielding the following relation between $L, K$ and $M$ :

$$
M=L+K-1 \text {. }
$$

The observation vector $\tilde{\mathbf{r}}_{k}$ in $k$ th sub-band can be written as a sub-vector of the observation at a given frequency band [17]. For each sensor, the expression of each observation sub-vector $\tilde{\mathbf{r}}_{k}$ can be written as:

$$
\tilde{\mathbf{r}}_{k}=\boldsymbol{\Lambda}_{k} \mathbf{A}_{1} \mathbf{D}^{k-1} \mathbf{c}+\tilde{\mathbf{n}}_{k}
$$

where $\mathbf{A}_{1}$ is made of the $L$ first rows of $\mathbf{A}, \boldsymbol{\Lambda}_{k}$ and $\tilde{\mathbf{n}}_{k}$ include the rows $\{k, k+1, \ldots, k+L-1\}$ of $\boldsymbol{\Lambda}$ and $\tilde{\mathbf{n}}$, respectively. $\mathbf{D}$ is the diagonal matrix which stands for the operator that shifts the observation on the corresponding sub-band between the different sub-bands, defined by: $\mathbf{D}=\operatorname{diag}\left(e^{-2 i \pi \Delta f \tau_{1}}, e^{-2 i \pi \Delta f \tau_{2}}, \cdots, e^{-2 i \pi \Delta f \tau_{P}}\right)$ and $\Delta f=$ $\frac{f_{L}-f_{1}}{L-1}$.

The matrix $\boldsymbol{\Lambda}_{k}$ is used to whiten the observation vector:

$$
\mathbf{y}_{k}=\boldsymbol{\Lambda}_{k}^{-1} \tilde{\mathbf{r}}_{k}=\mathbf{A}_{1} \mathbf{D}^{k-1} \mathbf{c}+\boldsymbol{\Lambda}_{k}^{-1} \tilde{\mathbf{n}}_{k} .
$$

Thus,

$$
\begin{aligned}
E\left[\mathbf{y}_{k} \mathbf{y}_{k}^{+}\right]=\boldsymbol{\Gamma}_{k}= & \mathbf{A}_{1} \mathbf{D}^{k-1} \boldsymbol{\Gamma}_{c}\left(\mathbf{D}^{k-1}\right)^{+} \mathbf{A}_{1}^{+} \\
& +\boldsymbol{\Lambda}_{k}^{-1} \boldsymbol{\Gamma}_{\tilde{n}_{k}}\left(\boldsymbol{\Lambda}_{k}^{-1}\right)^{+}=\boldsymbol{\Gamma}_{k}^{\tilde{s}}+\boldsymbol{\Gamma}_{k}^{\tilde{n}}
\end{aligned}
$$




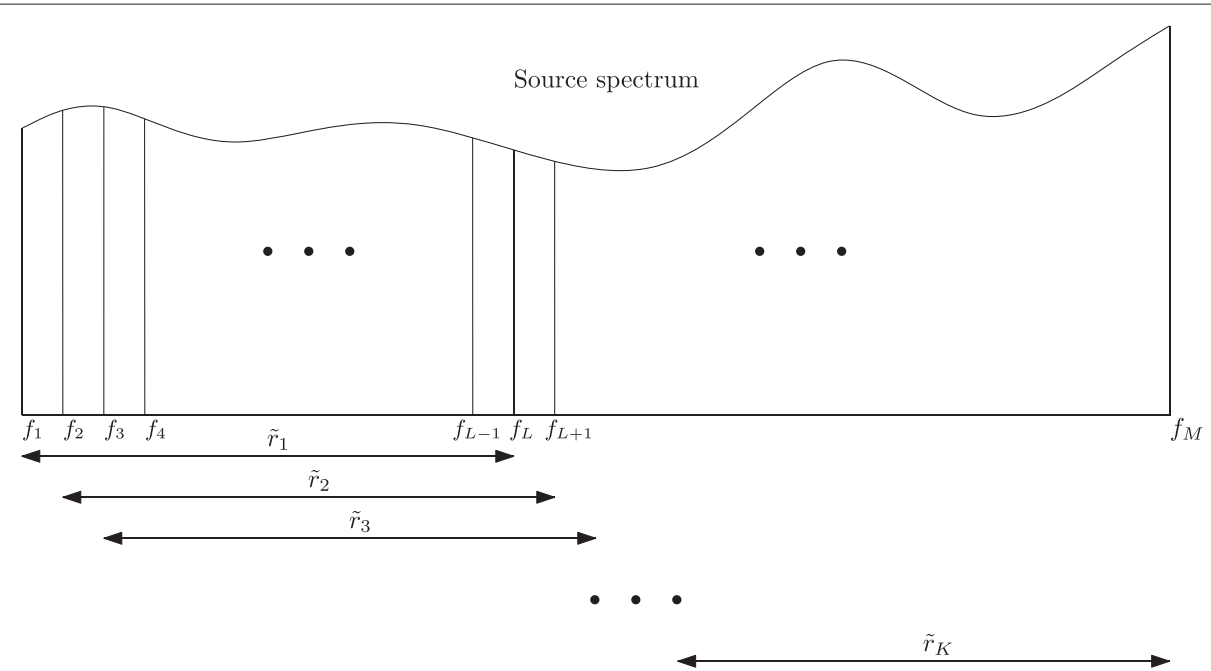

Figure 2 Sub-bands division used. The $M$ frequencies' band is divided into $K$ sub-bands of $L$ frequencies. The recovery between two consecutive bands is maximum $(L-1)$ frequencies.

where $\boldsymbol{\Gamma}_{c}=E\left[c c^{+}\right], \boldsymbol{\Gamma}_{k}^{\tilde{s}}=\mathbf{A}_{1} \mathbf{D}^{k-1} \boldsymbol{\Gamma}_{c}\left(\mathbf{D}^{k-1}\right)^{+} \mathbf{A}_{1}^{+}$and $\boldsymbol{\Gamma}_{k}^{\tilde{n}}=\boldsymbol{\Lambda}_{k}^{-1} \boldsymbol{\Gamma}_{\tilde{n}_{k}}\left(\boldsymbol{\Lambda}_{k}^{-1}\right)^{+}$. Let $\boldsymbol{\Gamma}_{m p}$ be the average of the different covariance matrices estimated at different subbands in the forward and backward directions. We have

$$
\boldsymbol{\Gamma}_{m p}=\frac{1}{2 K} \sum_{k=1}^{K}\left(\boldsymbol{\Gamma}_{k}+\mathbf{J} \boldsymbol{\Gamma}_{k}^{*} \mathbf{J}\right)=\boldsymbol{\Gamma}_{m p}^{\tilde{s}}+\boldsymbol{\Gamma}_{m p}^{\tilde{n}}
$$

where $\mathbf{J}$ stands for the anti-diagonal matrix of permutation that helps to generate the observation vector in the backward direction and $\boldsymbol{\Gamma}_{m p}^{\tilde{s}}=\frac{1}{2 K} \sum_{k=1}^{K}\left(\Gamma_{k}^{\tilde{s}}+\mathbf{J}\left(\boldsymbol{\Gamma}_{k}^{\tilde{s}}\right)^{*} \mathbf{J}\right)$ and $\boldsymbol{\Gamma}_{m p}^{\tilde{n}}=\frac{1}{2 K} \sum_{k=1}^{K}\left(\boldsymbol{\Gamma}_{k}^{\tilde{n}}+\mathbf{J}\left(\boldsymbol{\Gamma}_{k}^{\tilde{n}}\right)^{*} \mathbf{J}\right)$. High-resolution algorithms can be yielded using $\boldsymbol{\Gamma}_{m p}$ and $\mathbf{a}_{1}$ which is made of the $L$ first elements of $\mathbf{a}^{\prime}$.

This method will be used in the rest of this paper.

\section{Influence of the number of the sub-bands for a fixed sensor $j$}

To assess the decorrelation efficiency of this method, we will assume, for a given sensor, that there are two sources characterized by their amplitudes $c_{1}$ and $c_{2}$. Let $\gamma$ be their correlation coefficient that we define using the elements of the matrix $\boldsymbol{\Gamma}_{c}$ :

$$
\gamma=\frac{\boldsymbol{\Gamma}_{c}(1,2)}{\sqrt{\boldsymbol{\Gamma}_{c}(1,1) \boldsymbol{\Gamma}_{c}(2,2)}}
$$

where $\boldsymbol{\Gamma}_{c}(i, j)=E\left[c_{i} c_{j}^{*} y\right]$. For totally correlated sources, its modulus reaches 1 .

Let $\boldsymbol{\Gamma}_{c}^{K}=\frac{1}{2 K} \sum_{k=1}^{K} \mathbf{D}^{k-1} \boldsymbol{\Gamma}_{c}\left(\mathbf{D}^{k-1}\right)^{+}$and $\boldsymbol{\Gamma}_{J c J}^{K}$ so that $\mathbf{A}_{1} \boldsymbol{\Gamma}_{J c}^{K} \mathbf{A}_{1}^{+}=\frac{1}{2 K} \sum_{k=1}^{K} \mathbf{J} \boldsymbol{\Gamma}_{k}^{*} \mathbf{J}$. Then, Eq. (17) can be written as : $\boldsymbol{\Gamma}_{m p}=\mathbf{A}_{1}\left(\boldsymbol{\Gamma}_{c}^{K}+\boldsymbol{\Gamma}_{J J}^{K}\right) \mathbf{A}_{1}$.
$\Gamma_{c}^{K}$ 's $(1,2)$ element is

$$
\Gamma_{c}^{K}(1,2)=\frac{\Gamma_{c}(1,2)}{K} \sum_{k=1}^{K} e^{-2 i \pi \Delta f \Delta \tau(k-1)},
$$

where $\Delta \tau=\tau_{1}-\tau_{2}$. The correlation coefficient in $\boldsymbol{\Gamma}_{c}^{K}$ is $\gamma_{K}=\gamma\left(\frac{\sin (K \alpha)}{K \sin (\alpha)}\right) e^{-i(K-1) \alpha}$, where $\alpha=\pi \Delta f \Delta \tau$. The modulus of $\gamma_{K}$ is $\left|\gamma_{K}\right|=|\gamma|\left|\frac{\sin (K \alpha)}{K \sin (\alpha)}\right|$.

The term $\mathbf{J} \boldsymbol{\Gamma}_{k}^{*} \mathbf{J}$ consists in a double mirror symmetry along the rows and the columns of the matrix $\Gamma_{k}^{*}$. Then, $\Gamma_{J c}^{K}$ s $(1,2)$ element is

$$
\Gamma_{J c J}^{K}(1,2)=\frac{\Gamma_{c}(1,2)^{*}}{K} \sum_{k=1}^{K} e^{2 i \pi\left(2 f_{1}+(2 k+L-2) \Delta f\right) \Delta \tau} .
$$

Thus, the new correlation coefficient $\gamma_{m p}$ for the MSSP method can be expressed [20]:

$$
\gamma_{m p}=\gamma \frac{\sin (K \alpha)}{K \sin (\alpha)}\left(e^{-i(K-1) \alpha}+\frac{\Gamma_{c}^{*}(2,1)}{\Gamma_{c}(2,1)} e^{-i \beta}\right),
$$

where $\beta=2 \alpha\left(2-L-\frac{K+1}{2}\right)-4 \pi f_{1} \Delta \tau$. Therefore, the modulus of $\gamma_{m p}$ is

$$
\begin{aligned}
\left|\gamma_{m p}\right|=\left|\gamma_{K}\right| \mid \cos \left(\arg \left(c_{2}\right)-\arg \left(c_{1}\right)\right. & +\alpha(2-L-K) \\
& \left.-2 \pi f_{1} \Delta \tau\right) \mid .
\end{aligned}
$$

This new modulus $\left|\gamma_{m p}\right|$ is smaller than the original $|\gamma|$ and decreases as $K \Delta f \Delta \tau$ increases.

The size of the matrix $\boldsymbol{\Gamma}_{m p}$ must be at least $(P+1) \times$ $(P+1)$, which means $L \geq P+1$. On the other hand, as the sources are correlated and according to the algebra properties, the mean must be made on at least $P$ matrices 
$[18,26]$, which means $K \geq P$ and $M \geq 2 P+1$. If the forward and backward directions are used [27], this amount is reduced to $\frac{3 P}{2}$.

\section{Whitening of the modified data}

The high-resolution algorithms of array processing assume that the matrix $\boldsymbol{\Gamma}_{\tilde{n}}$ (see Eq. (17)) is diagonal. The noise covariance matrix $\Gamma_{m p}^{\tilde{n}}$ must then be $\sigma_{m p}^{2} \mathbf{I}_{L}$. Assuming $\boldsymbol{\Gamma}_{\tilde{n}}=\sigma^{2} \mathbf{I}_{M}$ gives

$$
\begin{aligned}
\boldsymbol{\Gamma}_{k}^{\tilde{n}} & =\boldsymbol{\Lambda}_{k}^{-1} \boldsymbol{\Gamma}_{\tilde{n}_{k}}\left(\boldsymbol{\Lambda}_{k}^{-1}\right)^{+} \\
& =\operatorname{diag}\left(\frac{\sigma^{2}}{\left|\tilde{s}\left(f_{k+l}\right)\right|^{2}}\right) ; l=\{0, \cdots, L-1\}
\end{aligned}
$$

and then

$$
\begin{gathered}
\Gamma_{m p}^{\tilde{n}}=\operatorname{diag}\left(\frac{\sigma^{2}}{2 K}\left(\sum_{k=1}^{K} \frac{1}{\left|\tilde{s}\left(f_{k+l}\right)\right|^{2}}+\frac{1}{\left|\tilde{s}\left(f_{k+L-1-l}\right)\right|^{2}}\right)\right) ; \\
\times l=0, \cdots, L-1 .
\end{gathered}
$$

The amount $\sum_{k=1}^{K} \frac{1}{\left|\tilde{s}\left(f_{k+l}\right)\right|^{2}}+\frac{1}{\left|\tilde{s}\left(f_{k+L-1-l}\right)\right|^{2}}$ varies with $l=0, \cdots, L-1$. Let $\Gamma_{m p}^{\tilde{n}}=\sigma^{2} \Sigma^{2}$, where $\boldsymbol{\Sigma}$ is the $L \times L$ diagonal matrix with $\boldsymbol{\Sigma}(l, l)=$ $\sqrt{\frac{1}{2 K}\left(\sum_{k=1}^{K} \frac{1}{\left|\tilde{s}\left(f_{k+l}\right)\right|^{2}}+\frac{1}{\left|\tilde{s}\left(f_{k+L-1-l}\right)\right|^{2}}\right)}$, Eq. (17) becomes

$$
\boldsymbol{\Gamma}_{m p}=\boldsymbol{\Gamma}_{m p}^{\tilde{r}}+\sigma^{2} \Sigma^{2}
$$

and let $\boldsymbol{\Gamma}_{w}$ be obtained from $\boldsymbol{\Gamma}_{m p}$ by the following transformation:

$$
\begin{aligned}
\boldsymbol{\Gamma}_{w}=\boldsymbol{\Sigma}^{-1} \boldsymbol{\Gamma}_{m p} \boldsymbol{\Sigma}^{-1} & =\boldsymbol{\Sigma}^{-1} \boldsymbol{\Gamma}_{m p}^{\tilde{r}} \boldsymbol{\Sigma}^{-1}+\sigma^{2} \boldsymbol{\Sigma}^{-1} \boldsymbol{\Sigma}^{2} \boldsymbol{\Sigma}^{-1} \\
& =\boldsymbol{\Sigma}^{-1} \boldsymbol{\Gamma}_{m p}^{\tilde{r}} \boldsymbol{\Sigma}^{-1}+\sigma^{2} \mathbf{I}_{L}
\end{aligned}
$$

The high-resolution methods must be slightly changed as the two subspaces have been shifted by $\Sigma^{-1}$. Rather than testing the vector $\mathbf{a}_{1}$ as presented in the previous section, the vector to be tested is $\mathbf{a}_{1}^{\prime}=\Sigma^{-1} \mathbf{a}_{1}$. Highresolution algorithms can now be used to estimate the TDOA on each sensor. In the following, we will present a way to localize the sources using the so-estimated TDOA.

\section{Source localization}

This section will address the localization issue. In the case of a linear antenna, the distance $\delta_{i, j}$ from source $i$, $i=1, \cdots, P$, to sensor $j, j=1, \cdots, N$, is, according to Al-Kashi theorem,

$$
\delta_{i, j}=\sqrt{\rho_{i}^{2}+((j-1) d)^{2}+2(j-1) d \rho_{i} \sin \left(\theta_{i}\right)},
$$

where $\rho_{i}$ and $\theta_{i}$ denote the range and DOA of the source against the antenna, as shown in Figure 3 , and $d$ is the distance between two consecutive sensors.
The presented method estimates the different TDOA $\hat{\tau}_{i, j}$, which correspond to $\hat{\tau}_{i, j}=\frac{\delta_{i, j}}{v}+T_{i}$, where $T_{i}$ is relative to each source $i$, as shown in Figure 4 , and $v$ is the wave velocity.

\section{Source links with estimated TDOA}

The estimated $\hat{\tau}_{i, j}$ are not sorted by source on each sensor. To localize the sources, it is important to know to which source each $\hat{\tau}_{i, j}$ is linked. This is not the case here, as shown in Figure 5 . So each $\hat{\tau}_{i, j}$ must be associated with the corresponding source.

To achieve that, we are looking for an indicator that will help us regroup the TDOA by source. In the following, we present a hierarchical clustering procedure that yields the TDOA sets.

Rather than considering the TDOA themselves, we will compare them. Thus, we propose to introduce the amount $O_{i, j}^{k, l}:$

$$
\begin{aligned}
O_{i, j}^{k, l} & =\frac{\hat{\tau}_{i, j}^{2}-\hat{\tau}_{k, l}^{2}-\left(j^{2}-l^{2}-2(j-l)\right)\left(\frac{d}{v}\right)^{2}}{2 d(j-l)} \\
& =o_{i, j}^{k, l}+\frac{T_{i}^{2}-T_{k}^{2}+2 \frac{\delta_{i, j}}{v} T_{i}-2 \frac{\delta_{k, l}}{v} T_{k}}{2 d(j-l)}
\end{aligned}
$$

where

$$
\begin{aligned}
o_{i, j}^{k, l} & =\frac{\left(\frac{\delta_{i, j}}{v}\right)^{2}-\left(\frac{\delta_{k, l}}{v}\right)^{2}-\left(j^{2}-l^{2}-2(j-l)\right)\left(\frac{d}{v}\right)^{2}}{2 d(j-l)} \\
& =\frac{\rho_{i}^{2}-\rho_{k}^{2}+2(j-1) d \rho_{i} \sin \left(\theta_{i}\right)-2(l-1) d \rho_{k} \sin \left(\theta_{k}\right)}{2 d(j-l) v^{2}}
\end{aligned}
$$

which becomes, in the case where $i=k$, that is, where $\hat{\tau}_{i, j}$ and $\hat{\tau}_{k, l}$ are the TDOA of the signals emitted by the same source:

$$
o_{i, j}^{i, l}=\frac{\rho_{i} \sin \left(\theta_{i}\right)}{v^{2}}
$$

and so,

$$
O_{i, j}^{i, l}=\frac{\rho_{i} \sin \left(\theta_{i}\right)}{v^{2}}+\frac{\left(\delta_{i, j}-\delta_{i, l}\right) T_{i}}{d(j-l) v} .
$$

For a given sensor $j \in[1, \cdots, N]$ and a given source $i \in[1, \cdots, P]$, we will consider $\left\{O_{i, j}^{k, l}, k \in[1, \cdots, P]\right\}$ $l \in[1, \ldots, j-1, j+1, \ldots, N]$. The right TDOA set corresponding to the $\hat{\tau}_{i, j}$ TDOA, $\left\{\hat{\tau}_{k_{i}^{l}, l}\right\}_{l \in[1, \ldots, j-1, j+1, \ldots, N]}$, will minimize the variance of $\left\{O_{i, j}^{k_{i}^{l}, l}\right\}_{l \in[1, \ldots, j-1, j+1, \ldots, N]}$. (see Figure 6). The indicator consists then for a given sensor and source to compute all the possible TDOA sets and choose the one that minimizes the variance of $\left\{O_{i, j}^{k, l}, k \in[1, \cdots, P]\right\}$ $l \in[1, \ldots, j-1, j+1, \ldots, N]$.

All the TDOA sets have now been identified. We can proceed to the source localization, which is the purpose of the next section. 


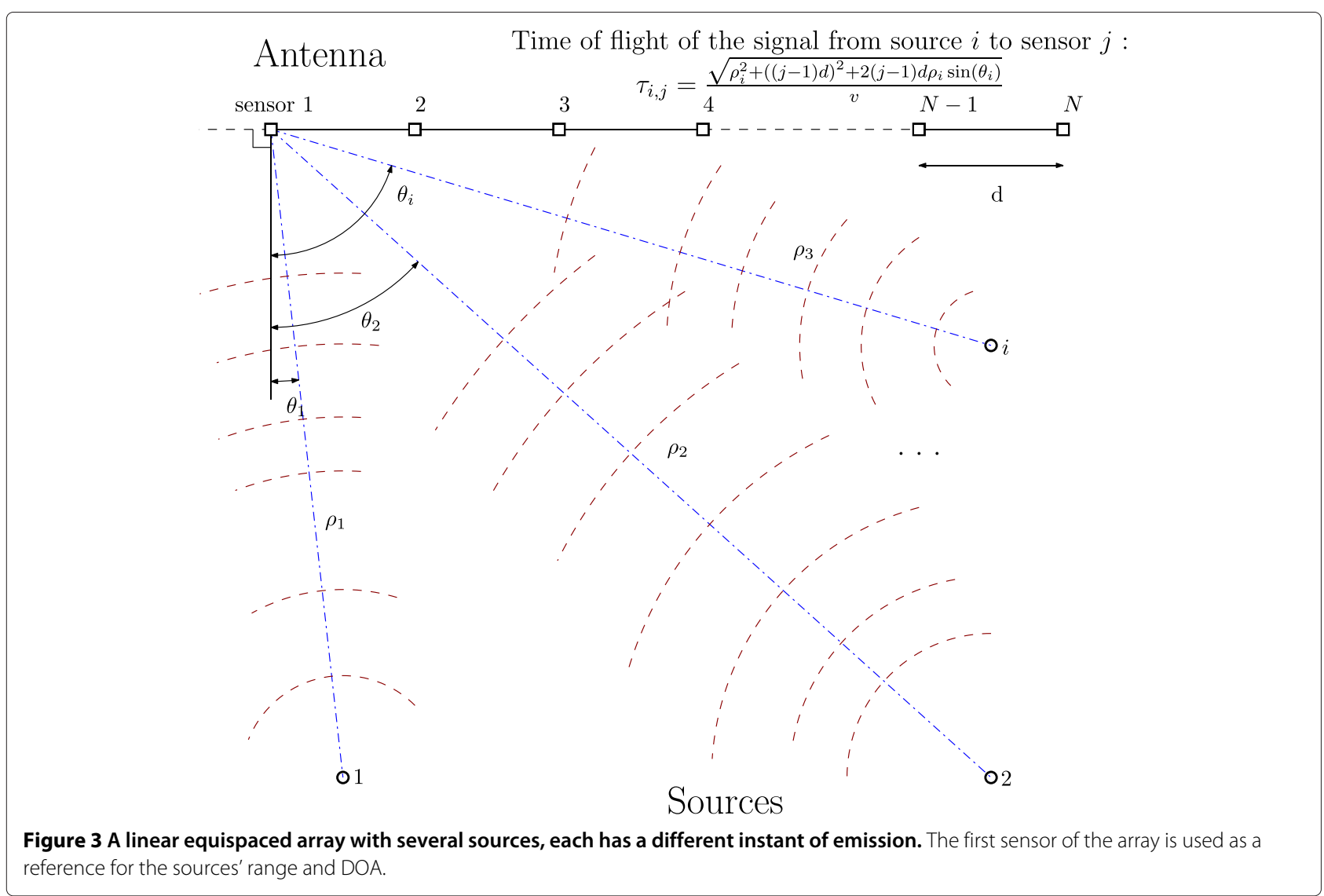

\section{Estimation of source range and DOA for a given source} In the following, we propose a method to estimate the ranges and DOA of the sources by using the estimated TDOA of the received signals on the different sensors. The proposed method is independent from the source to localize. We will get rid of superscript $i$ in order to simplify the notation.

For each TDOA set associated with the signal emitted by the $i$ th source and received on the sensors, we consider the following amount which evaluates the time delays between the first sensor and the other sensors of the antenna $(j=2, \cdots, N)$ :

$$
\hat{\tau}_{1}-\hat{\tau}_{j}=\frac{\delta_{1}-\delta_{j}}{v}=\frac{\delta_{1}}{v}\left(1-\sqrt{1+\frac{\delta_{j}^{2}-\delta_{1}^{2}}{\delta_{1}^{2}}}\right)
$$

using Eq. (27) we have $\delta_{i, 1}=\rho_{i}$.
In this section, index $i$ is omitted. Then, we set $\delta_{1}=\rho$. Let $h(j)=\frac{\delta_{j}^{2}-\delta_{1}^{2}}{\delta_{1}^{2}}=\frac{(j-1)^{2} d^{2}+2(j-1) d \rho \sin (\theta)}{\rho^{2}}$. Then, Eq. (32) becomes

$$
\left(\hat{\tau}_{1}-\hat{\tau}_{j}\right) v=\rho(1-\sqrt{1+h(j)})
$$

If $|h(j)|<1$ for all $j$; which is equivalent to the following assumption: $\frac{d(N-1)}{\sqrt{2}-1}<\rho$, indeed, $\forall j \in[2, \ldots, N],-1 \leq$ $h(j) \leq \frac{(N-1)^{2} d^{2}+2(N-1) d \rho}{\rho^{2}}$, then to have $\frac{(N-1)^{2} d^{2}+2(N-1) d \rho}{\rho^{2}}$ $<1$, we must ensure $d(N-1)(1+\sqrt{2})<\rho$; Eq. (33) can be expressed using the Taylor's development of $\sqrt{1+h(j)}$ and the Newton binomial formula:

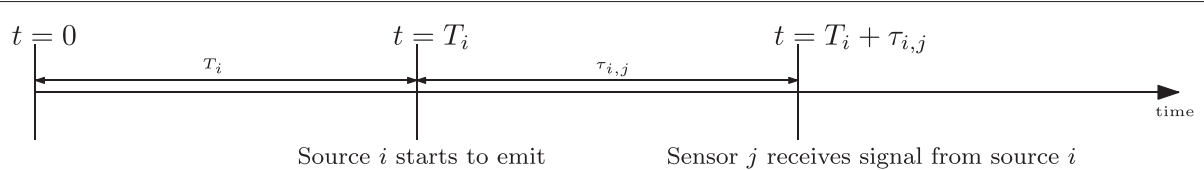

Figure $4 \boldsymbol{\tau}_{\boldsymbol{i}, \boldsymbol{j}}$ time needed for the signal from source $\boldsymbol{i}$ to reach the sensor $\boldsymbol{j} . T_{i}$ is time needed for the emitter's signal to reach the source $i$. 


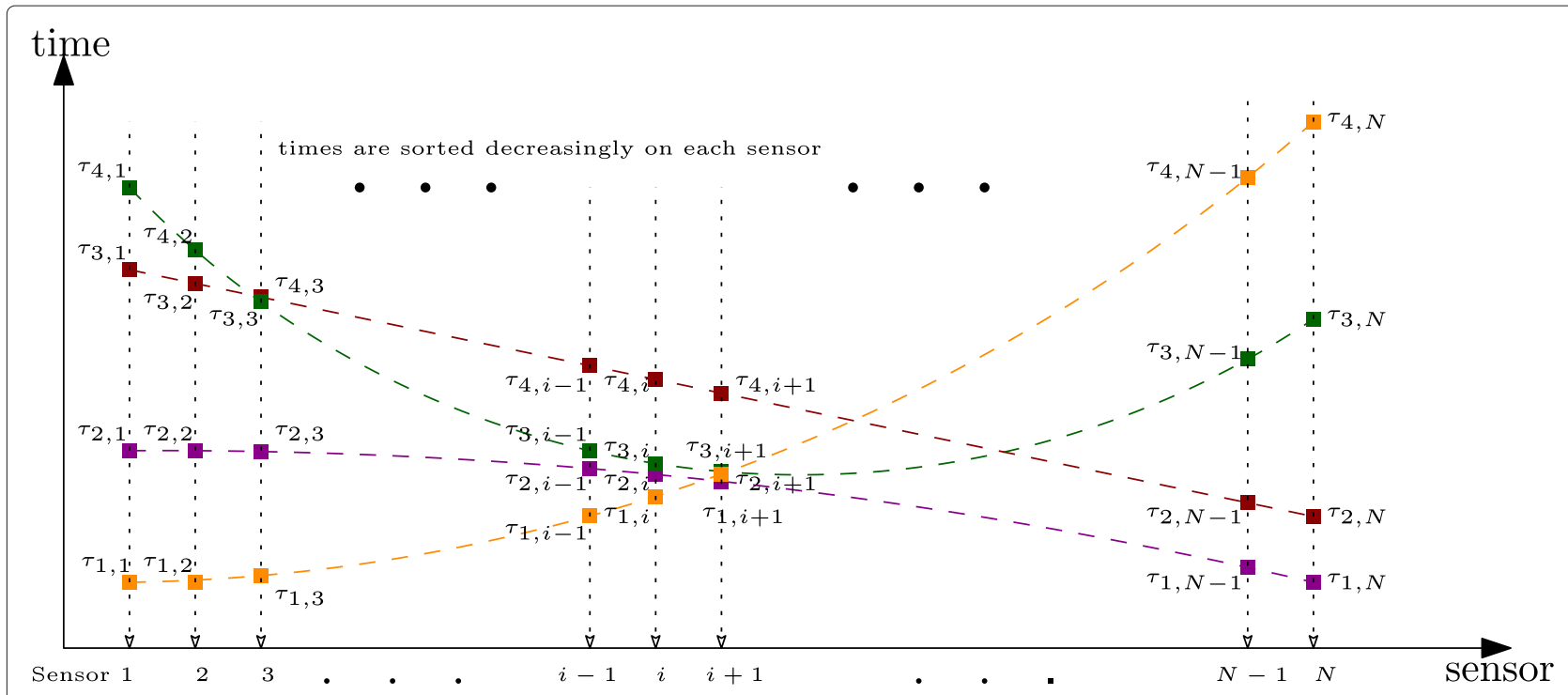

Figure 5 Example with four sources, and the evolution of different $\hat{\boldsymbol{\tau}}_{\boldsymbol{i}, \boldsymbol{j}}$. If the $\hat{\boldsymbol{\tau}}_{i, j}$ are only sorted in decreasing order on each sensor.

$$
\begin{aligned}
P(j)= & \left(\hat{\tau}_{1}-\hat{\tau}_{j}\right) v \\
= & \rho \sum_{n=1}^{\infty} \sum_{k=0}^{n}(-1)^{n} \frac{(2 n-2) !}{(n-1) ! 2^{n+k-1}(n-k) !}\left(\frac{d}{\rho}\right)^{n+k} \\
& \times \sin (\theta)^{n-k}(j-1)^{n+k} .
\end{aligned}
$$

The three first coefficients of this polynomial $P(j+1)=$ $A_{0}+A_{1} j+A_{2} j^{2}+A_{3} j^{3} \cdots+A_{n} j^{n} \cdots$ are $A_{0}=0, A_{1}=$ $-d \sin (\theta), A_{2}=\frac{d^{2}\left(\sin (\theta)^{2}-1\right)}{2 \rho}$. As $A_{0}=0$, we can consider $P^{\prime}(j)=\frac{P(j+1)}{j}=A_{1}+A_{2} j+A_{3} j^{2} \cdots+A_{n+1} j^{n} \cdots$.

With a number of sensors $N$ at least equal to 3 , it is possible to get a linear approximation of $P^{\prime}$ through a linear regression. This consists in estimating $A_{1}$ and

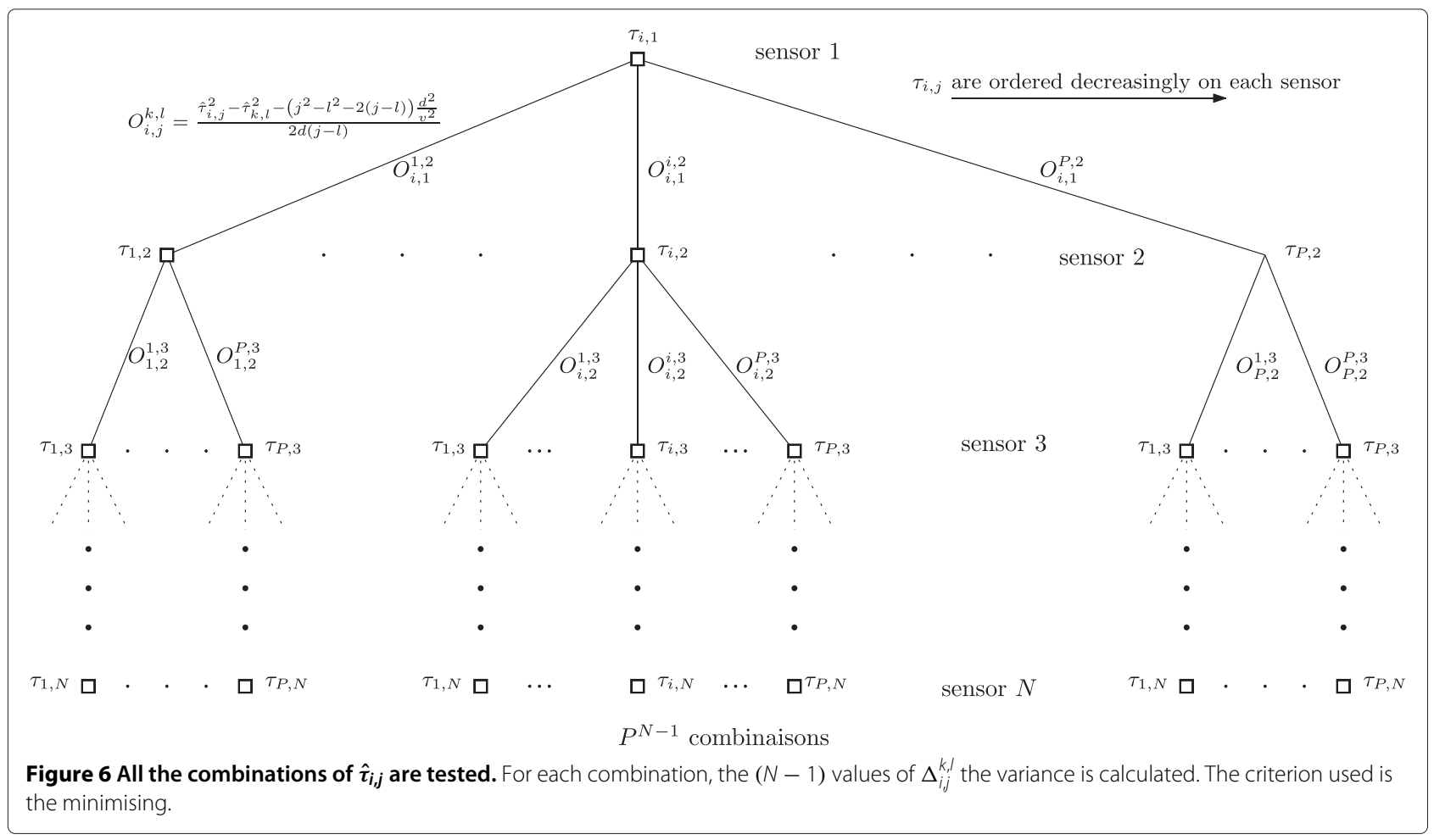


$A_{2}$. This is one approach to estimate the DOA and the range as:

$$
\hat{\theta}=\arcsin \left(\frac{\hat{A}_{1}}{d}\right)
$$

and

$$
\hat{\rho}=\frac{\hat{A}_{1}^{2}-d^{2}}{2 \hat{A}_{2}} .
$$

This simple estimation method suffers the fact that a bias on $\hat{\theta}$ induces a bias on $\hat{\rho}$ and this error mainly depends on the order at which the linear regression of $P^{\prime}$ is done.

In the next section, we present a method, to enhance this estimation using an iterative algorithm.

\section{Improvement of the source localization}

Range $\hat{\rho}$ and DOA $\hat{\theta}$ estimated in the previous section are used to initialize an iterative algorithm to improve the estimation accuracy, using a numerical solution to minimize a non-linear function with a set of parameters, like the Levenberg-Marquardt algorithm (LMA) [28].

Assuming that $x=\sin (\theta)$ and $y=\frac{1}{\rho}$, LMA will refine the estimation of $(x, y)$ by minimizing

$$
e(x, y)=\sum_{j=2}^{N}\left(K_{j}(x, y)\right)^{2},
$$

where $K_{j}(x, y)=\left(P^{\prime}(x, y, j)-\frac{\left(\hat{\tau}_{1}-\hat{\tau}_{j}\right) v}{j}\right) \forall 2 \leq j \leq N$ where $P^{\prime}(x, y, j)$ is, using Eq. (34), $P^{\prime}(x, y, j)=\frac{1}{j-1} \sum_{n=1}^{\infty}$ $\sum_{k=0}^{n}(-1)^{n} \frac{(2 n-2) !}{(n-1) ! 2^{n+k-1}(n-k) !} d^{n+k} y^{n+k-1} x^{n-k}(j-1)^{n+k}$.

The parameter vector $(x, y)$ is initialised by $x_{0}=\sin (\hat{\theta})$ and $y_{0}=\frac{1}{\hat{\rho}}$. At the $n$th iteration of LMA, $\left(x_{n}, y_{n}\right)$ is replaced by a new estimate $\left(x_{n+1}, y_{n+1}\right)=\left(x_{n}+\xi_{x_{n}}, y_{n}+\right.$ $\left.\xi_{y_{n}}\right)$. To determine $\left(\xi_{x_{n}}, \xi_{y_{n}}\right)$, the $K_{j}$ functions are linearised:

$$
\begin{aligned}
K_{j}\left(x_{n+1}, y_{n+1}\right)= & K_{j}\left(x_{n}+\xi_{x_{n}}, y_{n}+\xi_{y_{n}}\right) \approx K_{j}\left(x_{n}, y_{n}\right) \\
& +\boldsymbol{\Omega}(j-1,1) \xi_{x_{n}}+\boldsymbol{\Omega}(j-1,2) \xi_{y_{n}},
\end{aligned}
$$

where $\boldsymbol{\Omega}(j, l)$ is the $(j, l)$ element of the matrix $\boldsymbol{\Omega} . \boldsymbol{\Omega}$ is the $(N-1) \times 2$ Jacobian matrix of the $(N-1)$ derivatives of the functions $K_{j}, j=2, \cdots, N$ :

$$
\boldsymbol{\Omega}=\left(\begin{array}{cc}
\frac{\partial K_{2}}{\partial x} & \frac{\partial K_{2}}{\partial y} \\
\vdots & \vdots \\
\frac{\partial K_{j}}{\partial x} & \frac{\partial K_{j}}{\partial y} \\
\vdots & \vdots \\
\frac{\partial K_{N}}{\partial x} & \frac{\partial K_{N}}{\partial y}
\end{array}\right) .
$$

Using Eqs. (37) and (38), we obtain

$$
\begin{aligned}
e\left(x_{n+1}, y_{n+1}\right)=\sum_{j=2}^{N}\left(K_{j}\left(x_{n}, y_{n}\right)\right. & +\boldsymbol{\Omega}(j-1,1) \xi_{x_{n}} \\
& \left.+\boldsymbol{\Omega}(j-1,2) \xi_{y_{n}}\right)^{2} .
\end{aligned}
$$

In a matrix formalism, we obtain

$$
e\left(x_{n+1}, y_{n+1}\right)=\|\mathbf{K}+\mathbf{\Omega} \Xi\|^{2},
$$

where $\mathbf{K}=\left[K_{1}, \cdots, K_{j}, \cdots, K_{N}\right]^{T}$ and $\Xi=\left(\xi_{x_{n}}, \xi_{y_{n}}\right)^{T}$. Assume that $e\left(x_{n+1}, y_{n+1}\right)$ reaches its minimum, then its first derivative with respect to $\Xi$ is null. Equation (41) becomes in a matrix formalism:

$$
\begin{aligned}
& \left(\boldsymbol{\Omega}^{T} \boldsymbol{\Omega}\right) \Xi=\boldsymbol{\Omega}^{T} \mathbf{K} \\
& \Xi=\left(\boldsymbol{\Omega}^{T} \boldsymbol{\Omega}\right)^{-1} \boldsymbol{\Omega}^{T} \mathbf{K} .
\end{aligned}
$$

LMA consists in replacing Eq. (43) by a 'damped version', to avoid inverting an ill-conditioned matrix [28]:

$$
\begin{aligned}
& \left(\boldsymbol{\Omega}^{T} \boldsymbol{\Omega}+\lambda \operatorname{diag}\left(\boldsymbol{\Omega}^{T} \boldsymbol{\Omega}\right)\right) \Xi=\boldsymbol{\Omega}^{T} \mathbf{K} \\
& \Xi=\left(\boldsymbol{\Omega}^{T} \boldsymbol{\Omega}+\lambda \operatorname{diag}\left(\boldsymbol{\Omega}^{T} \boldsymbol{\Omega}\right)\right)^{-1} \boldsymbol{\Omega}^{T} \mathbf{K} .
\end{aligned}
$$

The (non-negative) damping factor $\lambda$ is adjusted at each iteration. If $e(x, y)$ decreases rapidly, a smaller value is used, bringing the algorithm closer to the Gauss-Newton algorithm, whereas if an iteration yields an insufficient decrease of the residual value, $\lambda$ can be increased, giving a step closer to the gradient descent direction [29]. LMA is stopped at a given step $n_{e}$ when the difference between $e\left(x_{n}, y_{n}\right)$ and $e\left(x_{n+1}, y_{n+1}\right)$ is less than a given threshold value. Then, $\theta$ and $\rho$ are estimated by $\hat{\theta}=\arcsin \left(x_{n_{e}}\right)$ and $\hat{\rho}=\frac{1}{y_{n_{e}}}$.

\section{Main algorithm}

\section{Steps}

We now afford the basic tools which are required for our algorithm. The different steps of the algorithm are enumerated as follows:

1. Sample the signal received on each sensor, and apply the FFT;

2. Then for each sensor:

(a) compute $\boldsymbol{\Gamma}_{m p}$ using Eq. (17);

(b) compute $\boldsymbol{\Gamma}_{w}$ using Eq. (26);

(c) estimate the number of sources $P$;

(d) use the modified high-resolution methods to estimate the TDOA : $\hat{\tau}_{1, j}, \ldots, \hat{\tau}_{P, j}$

3. build the TDOA sets using Eq. (28); 
DOA RMSE versus number of sources

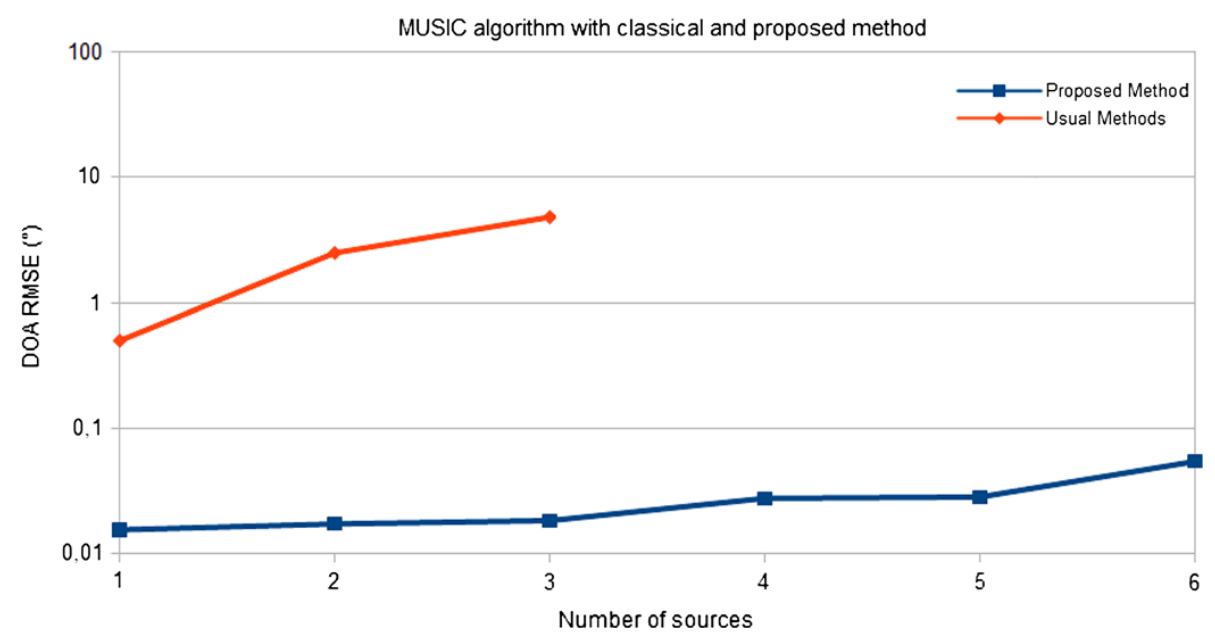

Figure 7 DOA RMSE $\left(^{\circ}\right)$ versus the number of sources $\mathrm{P}$ with MUSIC algorithm for both classical and proposed methods, with $\mathrm{SNR}=10 \mathrm{~dB}$.

4. with each TDOA set, obtain the DOA and the range of each source using the proposed method based on LMA.

\section{Parameters of interest}

The most influential parameter is the number $L$ of frequencies in the sub-bands:

1. It influences the performance of the MSSP decorrelation algorithm [19];

2. it specifies the dimension of the signal and noise subspaces, so it has to be higher than the number of sources $P$. Otherwise, these methods will not work [21];

3. as the number of sensors influences the spatial resolution and separation power of the methods [30-33]y, similarly $L$ influences on the time resolution and separation power for $\tau_{i, j}$ estimation;
The number of sensors $N$ :

1. It has to be larger than 3 to enable the polynomial fit and the iterative method to work, as the maximum degree for the polynomial fit is $N-1$;

2. if $N$ is too high, the recombination of the time series will not converge fast enough to be observed after a reasonable computation time.

The degree chosen for the polynomial fit presented:

1. It introduces a bias in $A_{1}$ and $A_{2}$ estimation and as a consequence in $\theta$ and $\rho$ estimation;

2. if $\theta \rightarrow \frac{\pi}{2}$ then $A_{2}$ tend to 0 , it might be of use to estimate and use $A_{3}=\frac{\rho}{2}\left(\frac{d}{\rho}\right)^{3}\left(\sin (\theta)-\sin ^{3}(\theta)\right)=$ $\frac{\rho}{2}\left(\frac{d}{\rho}\right)^{3} \sin (\theta) \cos ^{2}(\theta)$ for $\rho_{i}$ estimation.

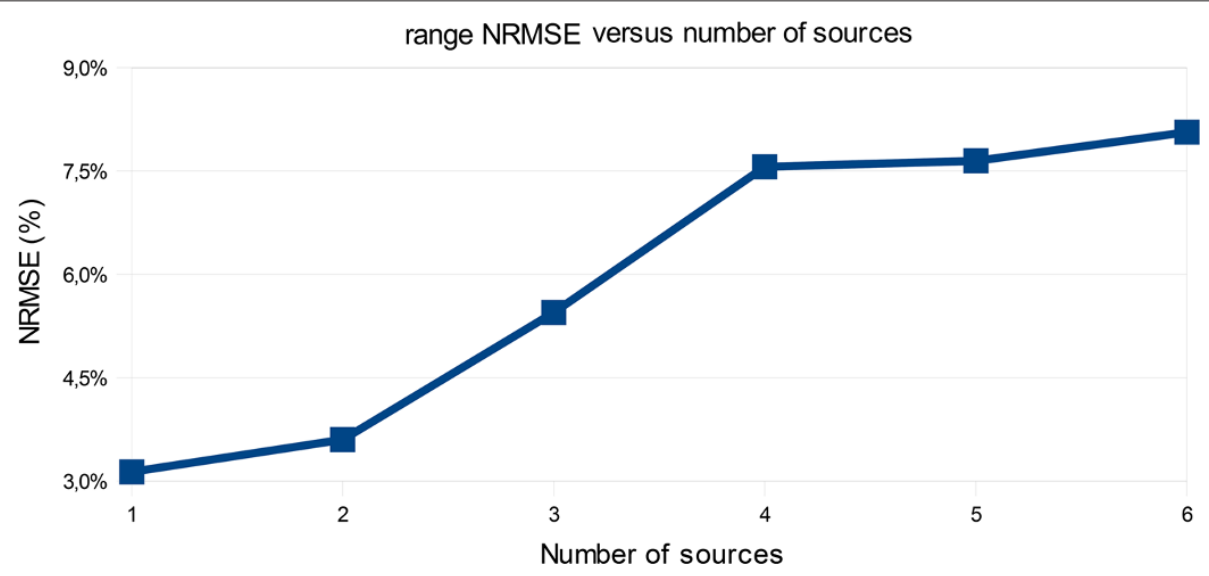

Figure 8 Range NRMSE (\%) versus the number of sources P with MUSIC algorithm for proposed method. 


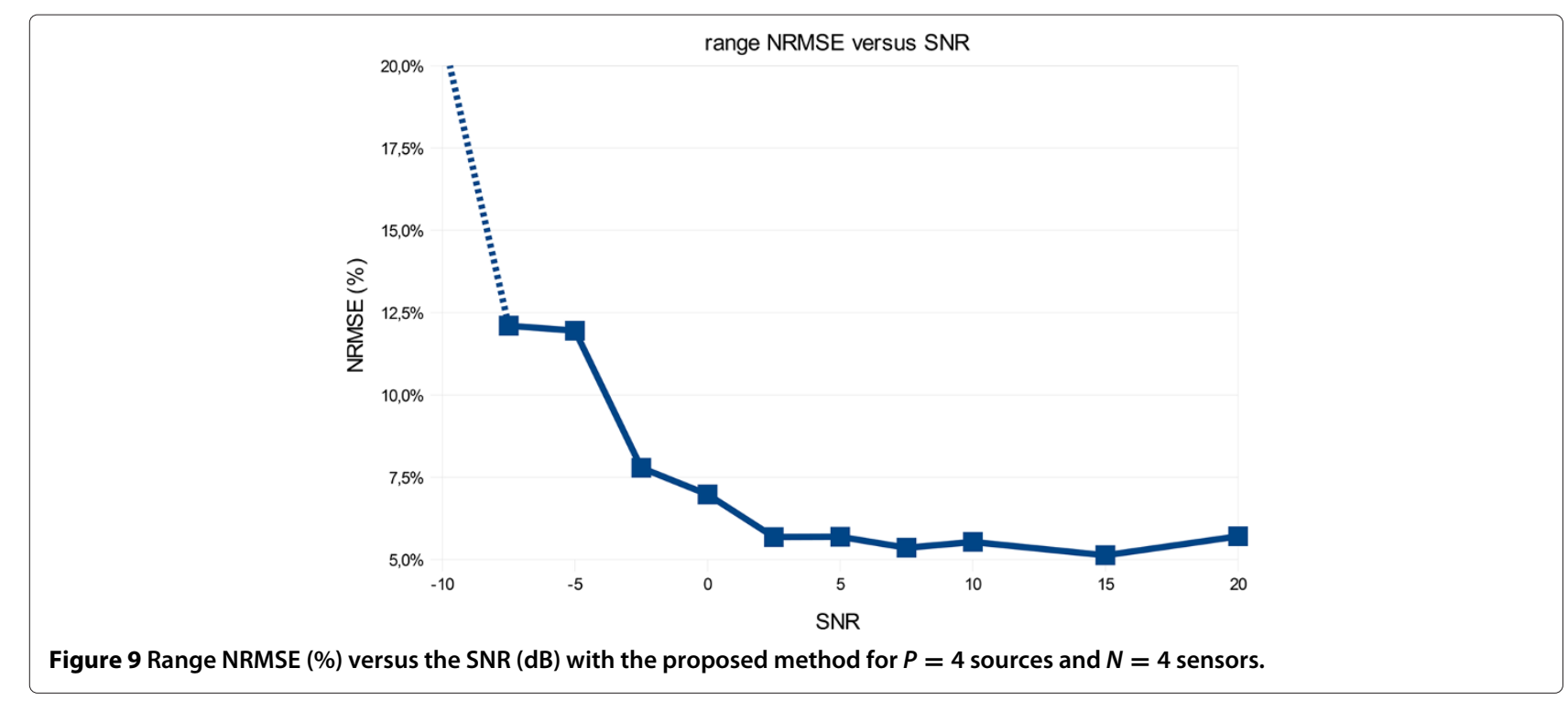

The total number of frequencies $M$ must be high enough so that:

1. The choice of $L$ value can be done adequately

2 . the number of sub-bands $K$ can be large enough to efficiently decorrelate the signals.

At last, let us consider the source space distribution. Respecting $d(N-1)(1+\sqrt{2})<\rho_{i}, \forall i=1, \cdots, P$, there is no limit in the choice of the different DOA values $\theta_{i}$. The only limitation appears while applying the high-resolution algorithm. Indeed, if the time estimation resolution is $\varepsilon_{H R}$, for all $i$, for all $k \neq i$ and for all $j$ we must have $\left|\tau_{i, j}-\tau_{k, j}\right|>$ $\varepsilon_{H R}$. Note that for all $n>1, A_{n} \propto \frac{1}{\rho^{n-1}}$, so $\lim _{\rho \rightarrow+\infty} A_{n}=0$ and especially $\lim _{\rho \rightarrow+\infty} A_{2}=0 . A_{1}$ is constant. Meaning that for a given antenna, as the range $\rho$ increases, as expected, it becomes more difficult to estimate it. The accuracy of $\theta$ estimation is not linked to $\rho$.

\section{Numerical results}

\section{Simulated data}

To localize immersed sources, we proposed to compare two methods: classical methods, based on a spatial analysis of the spatial covariance matrix of the data to estimate the DOA [5], and the proposed method, based on a spatiotemporal analysis which first estimates TDOA from the frequential covariance matrix of the data on each sensor and then estimates the DOA and range of the sources.

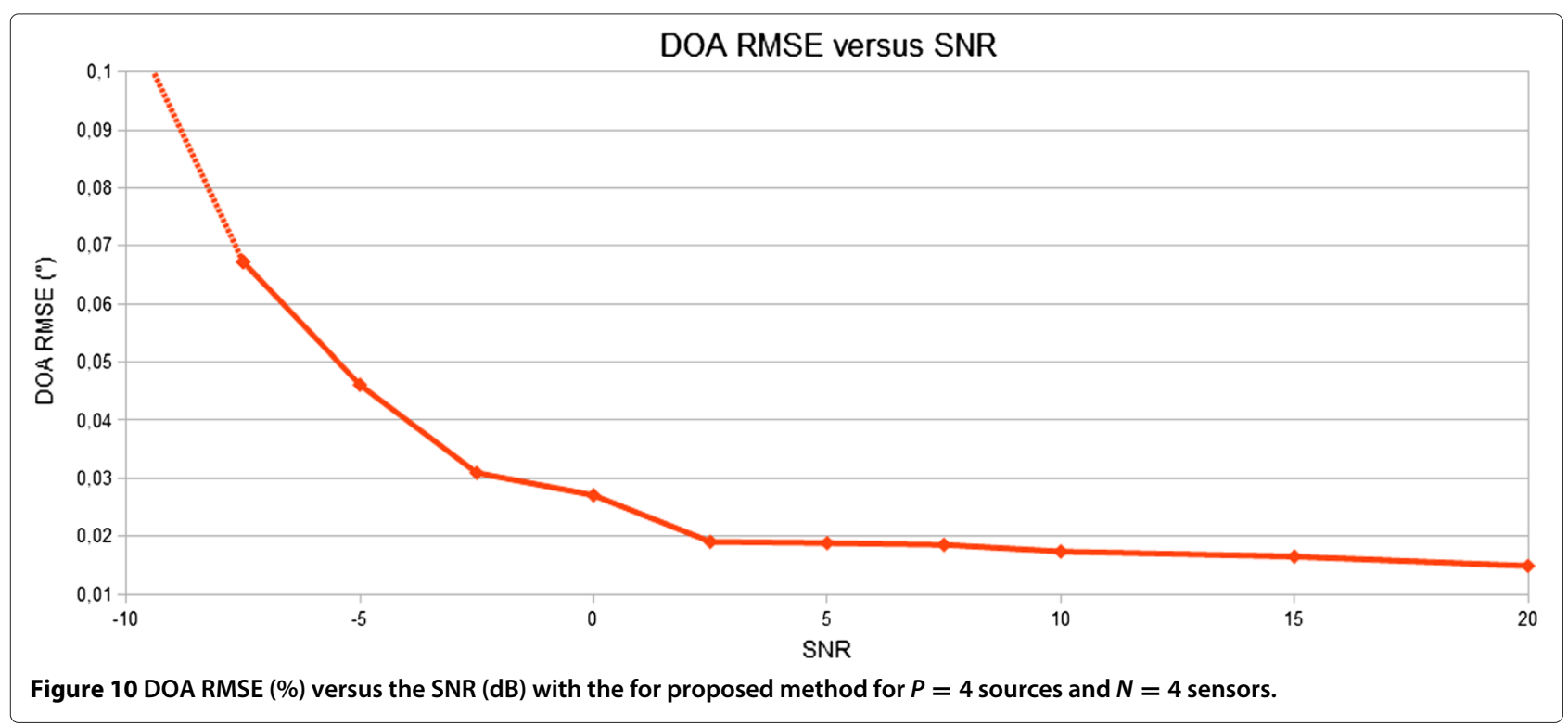




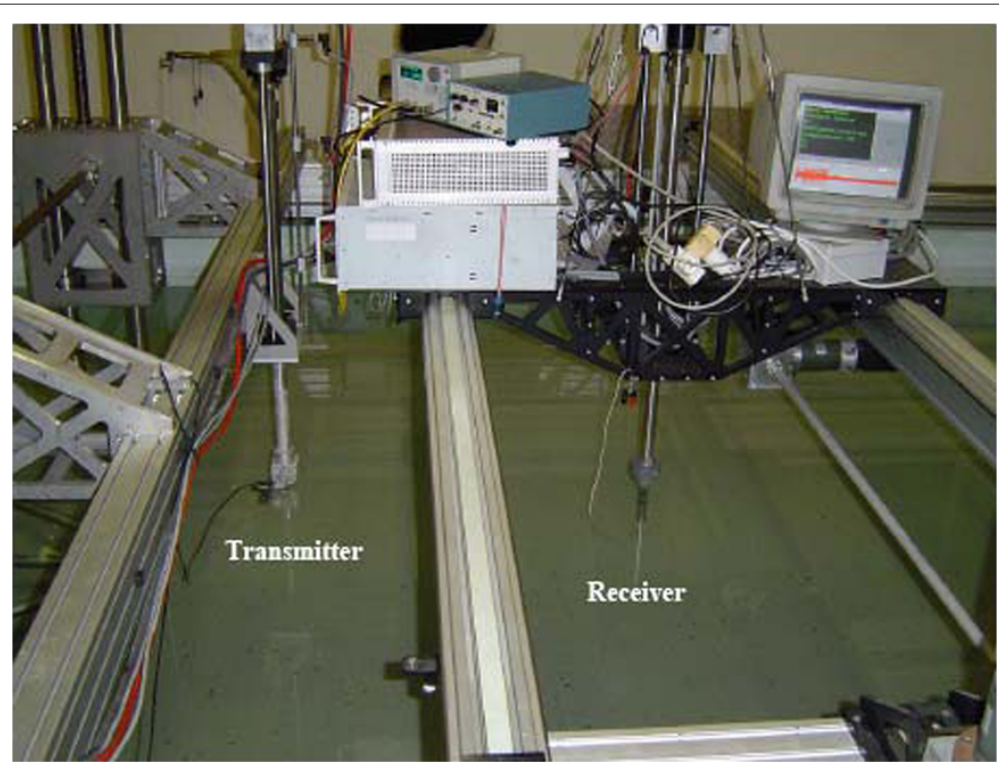

Figure 11 Experimental tank.

To decorrelate the signals, smoothing methods are used. Spatial smoothing for classical methods [18] and frequential smoothing for the proposed methods are used. The smoothing methods in spatial (respectively, frequential) domain require that the number of sensors $N$ (respectively, the number $M$ of frequencies) must be greater than or equal to $\frac{3 P}{2}$. As the number of sources increases, the classical methods will not give satisfying results when $P>$ $\frac{2 N}{3}$. The proposed method shifts all the spatial assumptions of classical methods into frequential assumptions.

We observed the performance of the classical and proposed methods when we increase gradually the number of sources from $P=1$ to 6 sources, whose ranges and DOA values are, in order of appearance, $\left(100 \mathrm{~m},-10^{\circ}\right.$; 98 $\left.\mathrm{m},-2.5^{\circ} ; 102 \mathrm{~m}, 2.5^{\circ} ; 96 \mathrm{~m},-5^{\circ} ; 104 \mathrm{~m}, 5^{\circ} ; 94 \mathrm{~m},-7.5^{\circ}\right)$. The signals are received on a rectilinear and of $N=4$ sensors which corresponds to a 1.5-m-length equispaced antenna. The received signals are simulated for an underwater acoustic experiment. Each source emits a linear chirp signal:

$$
s(t)=\left\{\begin{array}{cl}
e^{i 2 \pi\left(f_{0}+\frac{\Delta f}{2 T} \cdot t-\frac{\Delta f}{2}\right) t} & \text { if } 0 \leq t<T \\
0 & \text { else }
\end{array}\right.
$$

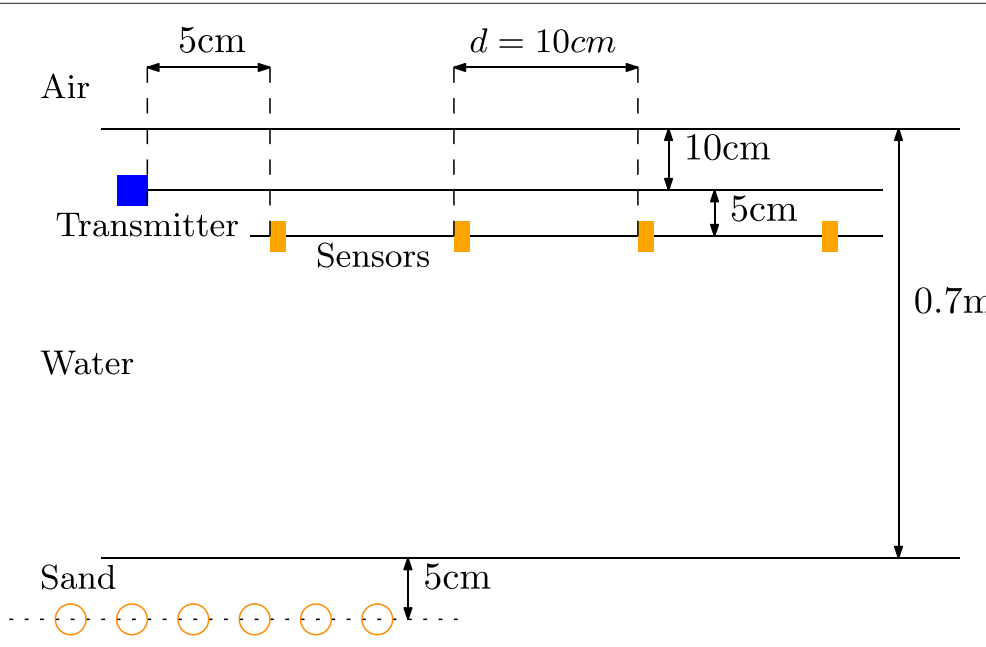

Objects

Figure 12 Experimental setup. 


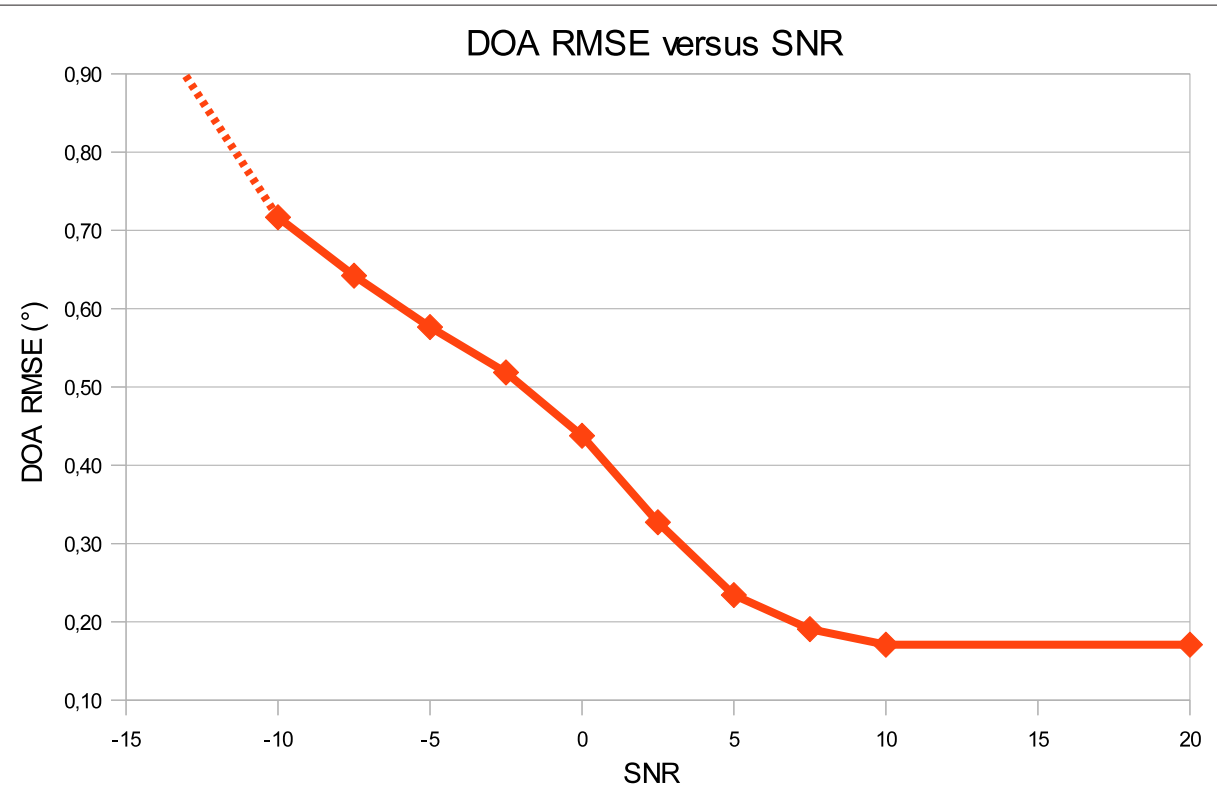

Figure 13 DOA RMSE $\left(^{\circ}\right)$ for experimental data, $N=4, P=5$, versus $S N R$.

with a span of $T=0.25 \mathrm{~s}$, a band of $\Delta f=3 \mathrm{kHz}$ and a central frequency of $f_{0}=1.5 \mathrm{kHz}$. The received signal on each sensor is generated using $r_{j}(t)=\sum_{i=1}^{P} c_{i, j} s\left(t-\tau_{i, j}\right)+$ $n_{j}(t) ; j=1, \cdots, 4$, where the noise $n_{j}(t)$ is white and Gaussian with variance $\sigma^{2}$ and the $c_{i, j}$ coefficients are randomly chosen and uniformly distributed so $\left|c_{i, j}\right|=1$. As the medium is assumed to be water, the velocity of the wave is set to $v=1,500 \mathrm{~m} / \mathrm{s}$ and the $\tau_{i, j}$ are calculated using Eq. (27). The SNR value is set to SNR $=10 \mathrm{~dB}$ defined by $\mathrm{SNR}=10 \log \left(\frac{|s|^{2}}{\sigma^{2}}\right)$.The received signal is sampled at $10 \mathrm{kHz}$.
For each simulation, $N_{t}=500$ trials are used, and $K=$ 150 sub-bands containing $L=50$ frequencies are used.

The DOA estimation root mean square error (RMSE) defined as:

$$
\operatorname{RMSE}(\theta)=\sqrt{\frac{1}{P} \sum_{i=1}^{P} \frac{1}{N_{t}} \sum_{j=1}^{N_{t}}\left[\left(\theta_{i}-\hat{\theta}_{i}^{j}\right)^{2}\right]},
$$

and the range normalized root mean square error (NRMSE) defined as:

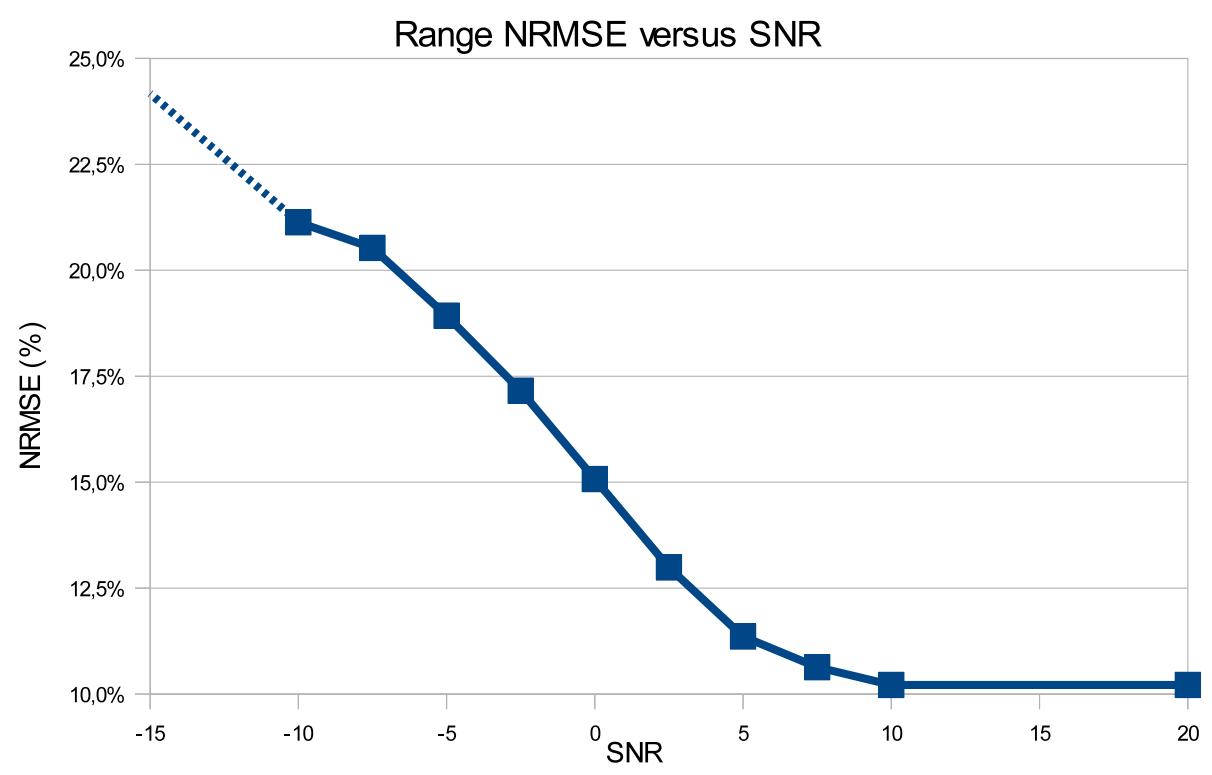

Figure 14 Range NRMSE (\%) for experimental data, $N=4, P=5$ versus SNR. 


$$
\operatorname{NRMSE}(\rho)=\sqrt{\frac{1}{P} \sum_{i=1}^{P} \frac{1}{N_{t}} \sum_{j=1}^{N_{t}}\left[\left(\frac{\rho_{i}-\hat{\rho}_{i}^{j}}{\rho_{i}}\right)^{2}\right]}
$$

are shown in Figures 7 and 8 versus the number of sources. When $P<N$, both methods are able to estimate the DOA, the proposed method giving more accurate results. When $P \geq N$, classical methods cannot be used. The proposed method still works with a satisfying accuracy .

Moreover, the proposed method estimates the range of the different sources, as shown in Figure 8. Figures 9 and 10 show the range and DOA RMSE versus the SNR.

\section{Experimental data}

In order to assess the efficiency of the proposed method, we propose to localize buried objects in a real-world environment. The experiment is carried out in an acoustic tank under conditions which are similar to those in an underwater environment. The bottom of the tank is filled with sand. The experimental device is presented in Figure 11. The tank is topped by two mobile carriages. The first carriage supports an issuer transducer and the second supports a receiver transducer managed by the computer. The observed signals come from various reflections on the objects being in the tank. In this experiment, we have recorded the reflected signals by a single receiver. This receiver is moved along with a straight line with a step $d=10 \mathrm{~cm}$ in order to create a uniform linear array of $N=5$ sensors. The buried objects are $P=6$ small cylindrical shells, buried at the same depth in sand, with DOA $\left\{32^{\circ}, 33^{\circ}, 34^{\circ}, 35^{\circ}, 36^{\circ}, 37^{\circ}\right\}$. The wave speed in the water $v_{1}=1,500 \mathrm{~m} / \mathrm{s}$ and in the sediment $v_{2}=1,700 \mathrm{~m} / \mathrm{s}$. Figure 12 sums up the experimental set-up.

For each experiment, the transmitted signal is a short pulse with a duration of $15 \mu \mathrm{s}$, the frequency band is $[150,250] \mathrm{kHz}$. At each sensor, time-domain data corresponding only to target echoes are collected with SNR equal to $20 \mathrm{~dB}$. To simulate different SNR values, we add a simulated white Gaussian noise. In this study, the received signal from direct path of propagation is used to fulfil matrix $\boldsymbol{\Lambda}$.

The estimation of RMSE and NRMSE as given in Eqs. (47) and (48) is presented versus SNR values in Figures 13 and 14. From Figures 13 and 14, it can be seen that the proposed method could effectively estimate the bearings and ranges of the buried objects in the real-world data since the RMSE and NRMSE are low.

\section{Conclusions}

This paper describes a way to address the problem of scattering object localization when the usual methods cannot be applied, especially because this method allows the number of sources to be higher than the number of sensors since the emitted signal is wideband. It enables, for instance, to have antennas with less sensors. Furthermore, several sources can have the same DOA. Thus, the proposed method exploit the spectral information of the wideband received spectrum. The signal received on each sensor is treated independently, using high-resolution algorithms to estimate the TDOA of each scattered image of the emitted signal. Then, the DOA and the range are jointly estimated to localize the objects. Numerical results for both simulated and experimental data reveal the good performance of our method for DOA estimation as well as for range estimation.

\begin{abstract}
Abbreviations
AIC: Akaike information criterion; DFT: Discrete Fourier transform; DOA: Direction of arrival; EM: Electromagnetic; EVD: Eigenvalues decomposition; GPR: Ground-penetrating radar; LMA: Levenberg-Marquardt algorithm; MDL: Minimum description length; MSSP: Modified spatial smoothing processing; MUSIC: Multiple signal classification; NRMSE: Normalize root mean square error; RMSE: Root mean square error; SNR: Signal-to-noise ratio; SVD: Singular values decomposition; TDOA: Time delay of arrival.
\end{abstract}

\section{Competing interests}

The authors declare that they have no competing interests.

\section{Acknowledgements}

The authors would like to thank the editor and two anonymous referees for their comments and suggestions which improved the article greatly. This work was supported by the French Armaments Procurement Agency (DGA). The authors would like to thank also Dr. J. P. Sessarego, from the LMA (Laboratory of Mechanic and Acoustic), Marseille, France, for providing us with experimental data.

Received: 10 October 2012 Accepted: 13 July 2013

Published: 26 July 2013

\section{References}

1. X Dérobert, C Fauchard, P Côte, EL Brusq, E Guillanton, J Dauvignac, C Pichot, Step-frequency radar applied on thin road layers. J. Appl. Geophys. 47(34), 317-325 (2001)

2. AM Zoubir, S Member, IJ Chant, CL Brown, B Barkat, C Abeynayake, Signal processing techniques for landmine detection using impulse ground penetrating radar. IEEE Sensors J. 2, 41-51 (2002)

3. SS Man, A Ikuo, Signal processing of ground penetrating radar using spectral estimation techniques to estimate the position of buried targets. EURASIP J. Adv. Signal Process. 2003(12), 970543 (2003)

4. J Capon, High-resolution frequency-wavenumber spectrum analysis. Proc. IEEE. 57(8), 1408-1418 (1969)

5. RO Schmidt, Multiple emitter location and signal parameters estimation. IEEE Trans. Antennas Propagation. 34(3), 276-280 (1986)

6. F Sellone, Robust auto-focusing wideband DOA estimation. Signal Process. 86(1), 17-37 (2006)

7. M Wax, STie-Jun, T Kailath, Spatio-temporal spectral analysis by eigenstructure methods. Acoustics, Speech Signal Process., IEEE Trans. 32(4), 817-827 (1984)

8. H Wang, M Kaveh, Coherent signal-subspace processing for the detection and estimation of angles of arrival of multiple wide-band sources. IEEE Trans. Acoustics, Speech Signal Processs. 33(4), 823-831 (1985)

9. H Hung, M Kaveh, Focussing matrices for coherent signal-subspace processing. IEEE Trans. Acoustics, Speech Process. 36(8), 1272-1281 (1988)

10. S Valaee, P Kabal, Wideband array processing using a two-sided correlation transformation. IEEE Trans. Signal Process. 43, 160-172 (1995)

11. YS Yoon, L Kaplan, J McClellan, TOPS: new DOA estimator for wideband signals. IEEE Trans. Signal Process. 54(6), 1977-1989 (2006)

12. J Zhang, J Dai, Z Ye, An extended TOPS algorithm based on incoherent signal subspace method. Signal Process. 90(12), 3317-3324 (2010)

13. WK Ma, TH Hsieh, CY Chi, DOA estimation of quasi-stationary signals with less sensors than sources and unknown spatial noise covariance: a 
Khatri-Rao subspace approach. IEEE Trans. Signal Process. 58(4), 2168-2180 (2010)

14. L Lu, HC Wu, K Yan, S lyengar, Robust expectation-maximization algorithm for multiple wideband acoustic source localization in the presence of nonuniform noise variances. IEEE Sensors J. 11(3), 536-544 (2011)

15. S Marcos, Calibration of a distorted towed array using a propagation operator. J. Acoust. Soc. Am. 93(4), 1987-1994 (1993)

16. S Bourennane, M Frikel. Localization of the wideband sources with estimation of the antenna shape, in Proceedings of the 8th IEEE Signal Processing Workshop on Statistical Signal and Array Processing (Corfu, 24-26 June 1996), pp. 97-100

17. V Reddy, A Paulraj, T Kailath, Performance analysis of the optimum beamformer in the presence of correlated sources and its behavior under spatial smoothing. IEEE Trans. Acoustics, Speech Signal Process. 35(7), 927-936 (1987)

18. TJ Shan, M Wax, T Kailath, On spatial smoothing for direction-of-arrival estimation of coherent signals. IEEE Trans. Acoustics, Speech Signal Process. 33(4), 806-811 (1985)

19. D Grenier, E Bosse, Decorrelation performance of DEESE and spatial smoothing techniques for direction-of-arrival problems. IEEE Trans. Signal Process. 44(6), 1579-1584 (1996)

20. H Yamada, M Ohmiya, Y Ogawa, K Itoh, Superresolution techniques for time-domain measurements with a network analyzer. IEEE Trans. Antennas Propagation. 39(2), 177-183 (1991)

21. PS Naidu, Sensor Array Signal Processing. (CRC Press, Boca Raton, 2000)

22. G Bienvenu, L Kopp, Optimality of high resolution array processing using the eigensystem approach. IEEE Trans. Acoustics, Speech Signal Process. 31(5), 1235-1248 (1983)

23. R Kumaresan, D Tufts, Estimating the angles of arrival of multiple plane waves. IEEE Trans. Aerosp. Electron. Syst. AES-19, 134-139 (1983)

24. F Glangeaud, C Latombe, Identification of electromagnetic sources. Annales Geophysicae 1, 245-251 (1983)

25. H Akaike, A new look at the statistical model identification. IEEE Trans. Automatic Control. 19(6), 716-723 (1974)

26. R Williams, S Prasad, A Mahalanabis, L Sibul, An improved spatial smoothing technique for bearing estimation in a multipath environment. IEEE Trans. Acoustics, Speech Signal Process. 36(4), 425-432 (1988)

27. S Pillai, B Kwon, Forward/backward spatial smoothing techniques for coherent signal identification. IEEE Trans. Acoustics Speech Signal Process. 37, 8-15 (1989)

28. K Levenberg, A method for the solution of certain non-linear problems in least squares. Q. Appl. Math. 2, 164-168 (1944)

29. Å Björck: Numerical Methods for Least Squares Problems (Siam, Philadelphia, 1996)

30. G Bienvenu. Eigensystem properties of the sampled space correlation matrix, in Proceedings of the IEEE ICASSP '83. International Conference on Acoustics, Speech, and Signal Processing, Volume 8 (Boston, 14-16 April 1983), pp. 332-335

31. P Germain, A Maguer, L Kopp. Comparison of resolving power of array processing methods by using an analytical criterion, in Proceedings of the IEEE ICASSP'89. International Conference on Acoustics, Speech, and Signal Processing, Volume 4, (Glasgow, 23-26 May 1989), pp. 2791-2794

32. D Thubert, L Kopp. Measurement accuracy and resolving power of high resolution passive methods, in Proceedings of EUSIPCO, Volume 2, (The Hague, 2-5 September 1986), pp. 1037-1040

33. FWhite, Performance of Bayes-optimal Angle-of-arrival Estimators. Technical report (Lincoln Laboratory), Massachusetts Institute of Technology, Department of Electrical Engineering and Computer Science (1983)

doi:10.1186/1687-6180-2013-131

Cite this article as: Villemin et al:: Spatio-temporal-based joint range and angle estimation for wideband signals. EURASIP Journal on Advances in Signal Processing 2013 2013:131. 